\title{
Androgen Regulation of the Rat Keratinocyte Growth Factor (KGF/FGF7) Promoter
}

\author{
Claudia Fasciana, ${ }^{1}$ Angelique C. van der Made, Peter W. Faber, ${ }^{2}$ and Jan Trapman \\ Department of Pathology, Erasmus University Rotterdam, P.O. Box 1738, 3000 DR Rotterdam, The Netherlands
}

\author{
Received February 20, 1996
}

\begin{abstract}
Keratinocyte Growth Factor (KGF/FGF7) is a candidate andromedin in normal embryonic development of male accessory sex glands, such as the prostate and seminal vesicles. The expression of KGF mRNA and protein is androgen-responsive. To elucidate the regulation of expression of the KGF gene, we isolated the first two exons of the KGF gene and approximately $15 \mathrm{~kb}$ upstream sequences. The major transcription start site was mapped. It is preceded by a CAAT-box and a TATA-box. Transient transfections in LNCaP cells revealed that, upon treatment with the synthetic androgen R1881, KGF promoter activity is upregulated 6 to 11 fold, indicating androgen regulation of the KGF promoter in the region from position -1900 to -1200 . The longest construct (BH-pLuc: -4700 to +901 ) has a much higher basal activity than the shorter constructs, indicating that in the region -4700 to -2700 additional activating sequences are present. () 1996 Academic Press, Inc.
\end{abstract}

Keratinocyte Growth Factor (KGF or FGF7) is a member of the Fibroblast Growth Factor family. KGF is exclusively expressed in mesenchymal and stromal cells of many organs and has a specific mitogenic effect on epithelial cells $(1,2)$, which express the KGF-receptor, a splice variant of Fibroblast Growth Factor Receptor 2 (3). The expression patterns of KGF and its high-affinity receptor have led to the assumption that KGF is a mediator of mesenchymal-epithelial cellular interactions $(4,5)$.

During development of male accessory sex glands, androgen-driven interactions between the mesenchyme and the epithelium are essential (6). The mesenchyme is the target of androgens, which is consistent with the finding that functional androgen receptors, which mediate the actions of androgens, prenatally and perinatally can only be detected in mesenchymal cells of the prostate (7). The intracellular androgen receptor is a ligand-dependent transcription factor and a member of the steroid/thyroid hormone/retinoic acid receptor family $(8,9)$. Defects in androgen receptor production or function result in aberrant male sexual development (10). Since in prostate (11) and seminal vesicles (12) KGF is expressed in mesenchymal cells, and the expression of the KGF mRNA as well as of the protein is androgen-responsive (11), KGF is considered to be an important candidate andromedin in normal development of male accessory sex organs. The possible role of KGF in androgen-driven development has recently been investigated in in vitro organ culture experiments. Administration of a KGF-antibody to the growth medium of in vitro grown newborn mouse seminal vesicles (12) and rat ventral prostates (13), results in inhibited growth of these organs and in inhibition of the epithelial branching morphogenesis. When newborn seminal vesicles were grown in Testosterone (T)-depleted medium and subsequently KGF was added, growth was approx. 50\% compared to newborn seminal vesicles that were grown in medium $+\mathrm{T}$.

In this study we describe the initial characterization of the rat KGF promoter and its activation by androgens.

\footnotetext{
${ }^{1}$ Corresponding author. Fax: 31-10-4366660.

${ }^{2}$ Present address: Neurogenetics Center, Massachusetts General Hospital East, Charlestown, MA 02129.

The nucleotype sequences reported in this article have been submitted to the EMBL, Genbank, and DDBJ Nucleotype Sequence Databases under Accession Number X95743.
} 


\section{MATERIALS AND METHODS}

Screening of a rat genomic library. A rat genomic EMBL3 $\lambda$-phage library (courtesy of A.P.N. Themmen, Rotterdam) was screened with a $\left.{ }^{32} \mathrm{P}\right]-\mathrm{dATP}$ (Amersham Ltd., Amersham, United Kingdom (UK)) random-labeled KGF specific 5' cDNA PCR probe obtained by amplification on rat genomic DNA (primer A: 5' -CACAGATAGGAGGAGGCCCAT-3' primer B: 5'-GCTAGAACAGTTCACGCTCGT-3'(14)) under standard conditions (15). Positive plaques were picked, purified and DNA was isolated. DNA of each clone was subsequently digested with a panel of restriction enzymes and subjected to Southern blot analysis to generate a restriction map. Appropriate fragments were subcloned and sequenced.

Cell culture. LNCaP cells were cultured in RPMI 1640, supplemented with 5\% fetal calf serum (Boehringer Mannheim, Germany) and antibiotics. For transfection experiments, cells were grown in Dulbecco's Modification of Eagle's Medium supplemented with 5\% steroid-depleted (dextran-charcoal treated) fetal calf serum. For examination of androgen-driven promoter activation, the synthetic androgen R1881 (New England Nuclear, Boston, MA) was added to a final concentration of $1 \mathrm{nM}$.

Constructs for transient transfections. The human androgen receptor expression plasmid pARO was described previously (16). The promoterless plasmid pLuc, which was used for cloning of KGF promoter fragments in front of the Luc reporter gene, was derived from pSLA3 (17) by insertion of an oligonucleotide containing a multiple cloning site (MCS) in the HindIII and NcoI sites of pSLA3 (18). KGF-pLuc reporter plasmids were generated by ligation of appropriate fragments in the MCS of pLuc. Constructs contain the following KGF promoter fragments: BamHI/HpaI-pLuc (BH-pLuc): -4700 to +901; EcoRV/EcoRV/HpaI-pLuc (EEH-pLuc): -2700 to +901; EcoRV/HpaI-pLuc (EH-pLuc): -1900 to +901; SacI/HpaIpLuc (SH-pLuc): -1200 to +901; PvuII/HpaI-pLuc (PH-pLuc): -536 to +901; HindIII/HpaI-pLuc (HH-pLuc): -66 to +901.

Transient transfections. Transient transfections of LNCaP cells were performed according to the calcium phosphate precipitation method essentially as described (19), using $1 \times 10^{6}$ cells per $25 \mathrm{~cm}^{2}$ flask, $5 \mu \mathrm{g}$ of the appropriate KGF-pLuc construct and $2.5 \mu \mathrm{g}$ pARO. Supercoiled plasmid DNA for transfections were isolated over Qiagen Plasmid-tips (Qiagen, Hilden, Germany). After $4 \mathrm{hr}$ incubation with the precipitate, culture medium was removed and replaced by phosphate buffered saline (PBS), containing $15 \%$ glycerol for a $90 \mathrm{sec}$ incubation at room temperature. Subsequently, transfected cells were incubated in culture medium with or without R1881 for at least $24 \mathrm{hr}$. Transfections were performed at least four times, with two different isolates of the appropriate plasmids.

Luciferase assay. Cells were washed once with PBS and lysed in $300 \mu$ l lysis buffer (25 mM Tris-phosphate pH 7.8, 8 $\mathrm{mM} \mathrm{MgCl} 2,1 \mathrm{mM}$ DTT, $1 \%$ Triton X-100, 15\% glycerol). Subsequently, $100 \mu 1$ Luciferin (0.25 $\mu \mathrm{M})$ (Sigma, St. Louis, $\mathrm{MO}) / 0.25 \mu \mathrm{M}$ ATP was added to $100 \mu \mathrm{l}$ of each extract, and luciferase activity was measured in a LUMAC $2500 \mathrm{M}$ Biocounter (LUMAC, Landgraaf, The Netherlands).

Rapid Amplification of cDNA ends (RACE). The RACE protocol was performed on 5'-RACE-Ready cDNA (Clontech, Palo Alto, CA) according to the directions of the manufacturer. In brief, PCR $\left(45 \mathrm{sec} 94^{\circ} \mathrm{C}, 60 \mathrm{sec} 60^{\circ} \mathrm{C}, 2 \mathrm{~min} 72^{\circ} \mathrm{C}\right.$ for 30 cycles, followed by a 7 min extension at $72^{\circ} \mathrm{C}$ ) was performed on $5^{\prime}$ anchored cDNA using the supplied anchor primer and the KGF specific primer RACE 1: 5'-TACTCCTAGGTCATGGGCCTC-3'. PCR products were separated on a $2 \%$ agarose gel and blotted to a Hybond $\mathrm{N}^{+}$filter (Amersham Ltd., Amersham, UK) for Southern blot analysis. Filters were hybridized with a $\left.{ }^{32} \mathrm{P}\right]$-dATP random-labeled 504 bp HindIII-BamHI KGF promoter fragment, followed by washes to $1 \times$ $\mathrm{SSC} / 0.1 \% \mathrm{SDS}$ at $65^{\circ} \mathrm{C}$. Filters were exposed for autoradiography at $-80^{\circ} \mathrm{C}$.

\section{RESULTS}

\section{Isolation of the Rat KGF Promoter}

To isolate the promoter of the rat KGF gene, we screened a rat genomic library with a $240 \mathrm{bp}$ KGF cDNA probe (see Materials and Methods). One of the phage inserts isolated (11.2) contained the $5^{\prime}$ part of the KGF gene and approximately $15 \mathrm{~kb}$ upstream sequences. Figure 1a shows a restriction map of a region of clone 11.2 that spans approx. $6 \mathrm{~kb}$. By comparing the genomic sequences obtained from clone 11.2 to cDNA sequences, we found that, besides the three exons known so far of the KGF gene (20), an additional upstream exon is present (exon 1A). The size of intron $1 \mathrm{~A}$ is $585 \mathrm{bp}$. In the lower part of figure 1a the $5^{\prime}$ region of the KGF gene with a part of exon $1 \mathrm{~B}$, intron $1 \mathrm{~A}$ and exon $1 \mathrm{~A}$ is presented in more detail. Figure $1 \mathrm{~b}$ shows the sequences obtained from the $5^{\prime}$ region of the KGF gene. The exon/intron boundaries are according to consensus sequences.

To determine the exact $5^{\prime}$ boundary of exon $1 \mathrm{~A}$ and to map the transcription start site we performed Rapid Amplification of cDNA Ends (RACE) on 5'-RACE-ready rat kidney cDNA. Southern blot analysis of the product is depicted in Figure 1c. One specifically hybridizing band of approx. $400 \mathrm{bp}$ was detected. The fragment was cloned and the major transcription start site was mapped by sequencing (see Figure 1b). This start site is preceded by a CAAT box at position -51 


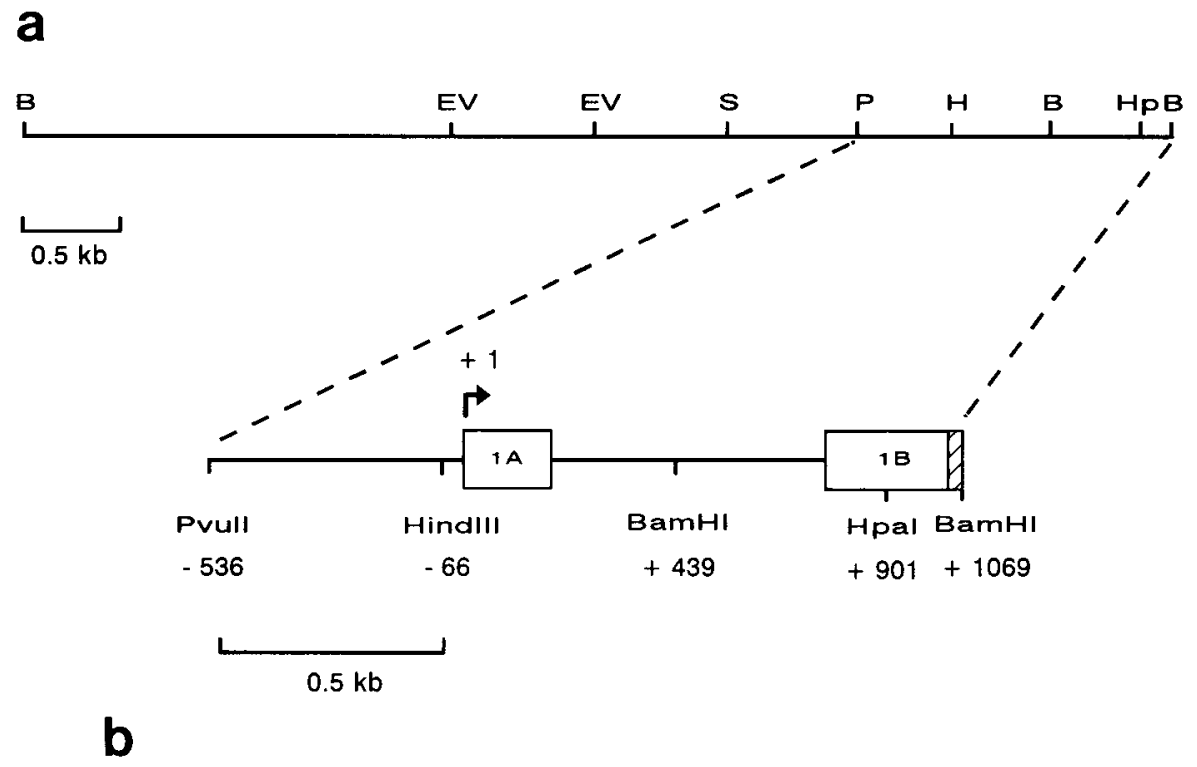

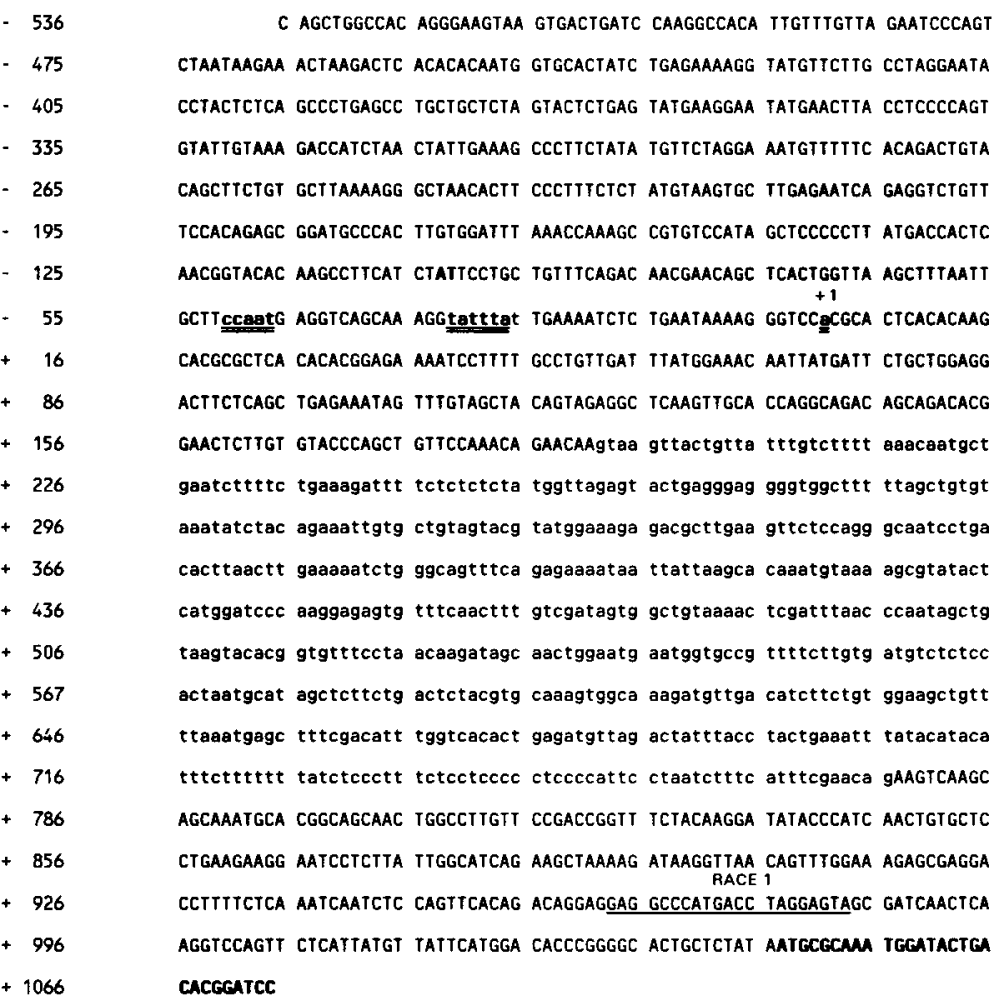

FIG. 1. Characterization of the rat KGF promoter. (a) Partial restriction map of the KGF promoter region. B, BamHI; EV, EcoRV; S, SacI, P, PvuII, HIII, HindIII, Hp, HpaI. In the lower part the organization of the $5^{\prime}$ part of the KGF gene is shown in more detail. Boxes represent exons. Untranslated cDNA is represented by an open box, translated sequences by a hatched box. The major transcription start site is indicated by the arrow. (b) Sequence of the 5' part of the KGF gene. Translated sequences are in bold letters. The transcription start site, CAAT and TATA box are in lower case letters and double underlined. Intron sequences are in lower case letters. The position of primer RACE 1 is underlined. (c) Southern blot analysis of KGF RACE cDNA. For experimental details see Materials and Methods. 


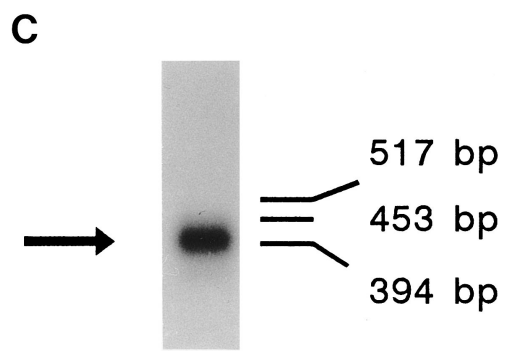

FIG. 1.-Continued.

and a TATA box at position -32 (Figure 1b). Some transcripts start at the A or the $\mathrm{T}$ at positions -103 and -102 respectively, but they represent a far minority of all cDNA fragments examined.

\section{The Rat KGF Promoter Is Androgen Responsive in Transient Transfections}

Deletion constructs of the KGF promoter hooked to the Luc reporter gene were transiently transfected LNCaP cells together with the androgen receptor expression plasmid pARO. We used a series of deletion constructs containing upstream sequences (see Materials and Methods). Figure 2 shows the results of the experiments. All KGF deletion constructs showed basal promoter activity compared to the "empty" luciferase reporter plasmid as a control, but the longest construct (BHpLuc: $-4700 /+901$ ) had a 5 to 15 fold higher basal activity than the shorter constructs. When cells were incubated in the presence of the synthetic androgen R1881, constructs HH-pLuc, PH-pLuc, and SH-pLuc did not show a marked induced Luc activity upon R1881 treatment. However a 6 to 11 fold induction of Luc activity was measured upon treatment with $1 \mathrm{nM}$ R1881 in the 3 longer constructs (BH-pLuc, EEH-pLuc and EH-pLuc). Although the androgen induction of BH-pLuc was lower than of the shorter constructs (6-fold compared to 11-fold), again, the absolute promoter activity was highest of all constructs. The drop in R1881 inducibility between EH-pLuc and SH-pLuc suggests that the region between positions -1900 and -1200 is involved in androgen regulation of the $\mathrm{KGF}$ promoter.

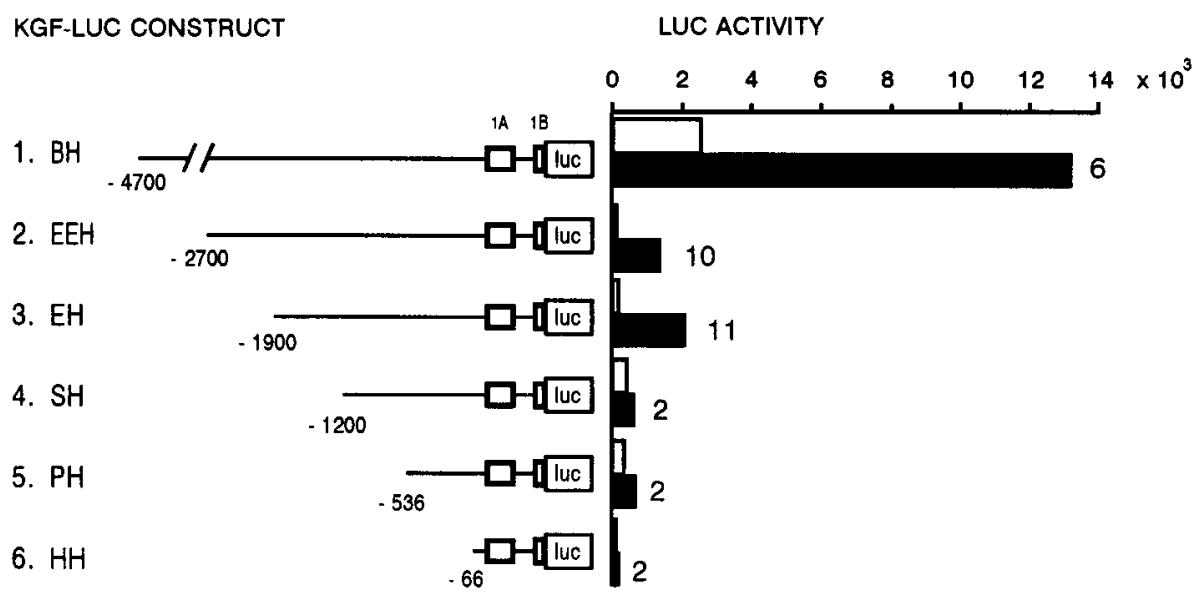

FIG. 2. Transient transfections of LNCaP cells with deletion constructs of the KGF promoter region and the human androgen receptor expression vector (pARO). KGF promoter fragments were cloned in front of the Luciferase reporter gene. Cells were grown in the absence (open bar) and presence of $1 \mathrm{nM} \mathrm{R} 1881$ (black bar). Relative induction factors are indicated at the top of the bars. 


\section{DISCUSSION}

KGF is considered to be a candidate andromedin in the development of male accessory sex glands (21). Our observations support this concept as we show that KGF promoter activity is regulated by androgens. In transient transfections, the region from position -1900 to -1200 is involved in this androgen regulation of the KGF promoter, which was confirmed when we cloned the genomic fragment in front of a minimal TK-promoter in a Luc reporter vector. Also in this setting Luc activity was upregulated after treatment with R1881 (data not shown). It can be presumed that the -1900 to -1200 fragment contains one or more androgen receptor binding sites. Further sequence analysis, combined with functional studies and in vitro protein-DNA interactions, may reveal the details of androgen regulation of the KGF promoter.

From the results of the transient transfections, presented in figure 2, it is clear that, although all KGF deletion constructs show basal activity, BH-pLuc $(-4700$ to +901$)$ is by far most active. Upon treatment with androgens, the induction of BH-pLuc is lower than that of the shorter constructs, but, again, the absolute promoter activity is much higher. This may mean, that in the -4700 to -2700 region additional activating sequences are present, as well as sequences, that modulate the androgen-induction.

Recently the human KGF promoter was isolated (22). Comparison of the sequences of the rat KGF promoter to the human promoter shows that the homology is high up to position -255 in the rat promoter (78\%), upstream of this point sequences diverge (data not shown). The human KGF promoter contains two transcription start sites, whereas in the rat KGF promoter we found one major transcription start site. The location of the major start site is identical in both genes. Both the human and the rat KGF promoter have a CAAT-box and a TATA-box upstream of the major transcription start site.

In this study we used $\mathrm{LNCaP}$ epithelial prostatic carcinoma cells for our transfection experiments, since LNCaP cells endogenously express the androgen receptor and can easily be transfected (18). Available rat mesenchymal (rUGM (23)) and stromal (Nbf-1 (24)) prostatic cell lines were not transfectable. The human KGF promoter could not be activated in a number of transfected epithelial cell lines tested, which were further shown not to express KGF mRNA (21). We have investigated endogenous KGF mRNA expression in LNCaP cells on a Northern blot and we could detect the $2.4 \mathrm{~kb}$ KGF transcript (data not shown).

\section{ACKNOWLEDGMENTS}

This study was supported by Grant 901-28-078 of the Dutch Society of Scientific Research (NWO) and by National Institutes of Health Grant DK 47596. We thank Frank van der Panne for excellent photowork and Axel P. N. Themmen for kindly providing us with the rat genomic library.

\section{REFERENCES}

1. Rubin, J. S., Osada, H., Finch, P. W., Taylor, W. G., Rudikoff, S., and Aaronson, S. A. (1989) Proc. Natl. Acad. Sci. USA 86, 802-806.

2. Finch, P. W., Rubin, J. S., Miki, T., Ron, D., and Aaronson, S. A. (1989) Science 245, 752-755.

3. Miki, T., Bottaro, D. P., Fleming, T. P., Smith, C. L., Burgess, W. H., Chan, A. M.-L., and Aaronson, S. A. (1992) Proc. Natl. Acad. Sci. USA 89, 246-250.

4. Finch, P. W., Cunha, G. R., Rubin, J. S., Wong, J., and Ron, D. (1995) Dev. Dynamics 203, $223-240$.

5. Rubin, J. S., Bottaro, D. P., Chedid, M., Miki, T., Ron, D., Cheon, H-G., Taylor, W. G., Fortney, E., Sakata, H., Finch, P. W., and LaRochelle, W. J. (1995) Cell Biol. Int. 19:5, 399-411.

6. Cunha, G. R., Donjacour, A. A., Cooke, P. S., Mee, S., Bigsby, R. M., Higgins, S. J., and Sugimura, Y. (1987) Endocrine Rev. 8:3, 338-362.

7. Cooke, P. S., Young, P., and Cunha, G. R. (1991) Endocrinol. 128:6, 2867-2873.

8. Mangelsdorf, D. J., Thummel, C., Beato, M., Herrlich, P., Schütz, G., Umesono, K., Blumberg, B., Kastner, P., Mark, M., Chambon, P., and Evans, R. M. (1995) Cell 83, 835-839.

9. Beato, M., Herrlich, P., and Schütz, G. (1995) Cell 83, 851-857.

10. McPhaul, M. J., Marcelli, M., Tilley, W. D., Griffin, J. E., and Wilson, J. D. (1991) FASEB J. 5, $2910-2915$. 
11. Yan, G., Fukabori, Y., Nikolaropoulos, S., Wang, F., and McKeehan, W. L. (1992) Mol. Endocrinol. 6:12, $2123-2128$.

12. Alarid, E. T., Rubin, J. S., Young, P., Chedid, M., Ron, D., Aaronson, S. A., and Cunha, G. R. (1994) Proc. Natl. Acad. Sci. USA 91, 1074-1078.

13. Sugimura, Y., Cunha, G. R., Hayward, S., Hayashi, N., Arima, K., Kawamura, J., Rubin, J., and Aaronson, S. (1993) Mol. Cell. Diff. 1, 423.

14. Yan, G., Nikolaropoulos, S., Wang, F., and McKeehan, W. L. (1991) In Vitro Cell. Dev. Biol. 27A, 437-438.

15. Sambrook, J., Fritsch, E. F., and Maniatis, T. (1989) Molecular Cloning: A Laboratory Manual. Cold Spring Harbor Laboratory Press, Cold Spring Harbor.

16. Brinkmann, A. O., Faber, P. W., van Rooij, H. C. J., Kuiper, G. G. J. M., Ris, C., Klaassen, P., van der Korput, J. A. G. M., Voorhorst, M. M., van Laar, J. H., Mulder, E., and Trapman, J. (1989) J. Steroid Biochem. 34, 307-310.

17. van Dijk, M. A., van Schaik, F. M. A., Bootsma, H. J., Holthuizen, P., and Sussenbach, J. S. (1991) Mol. Cell. Endocrinol. 81, 81-94.

18. Cleutjens, K. B. J. M., van Eekelen, C. C. E. M., van der Korput, H. A. G. M., Brinkmann, A. O., and Trapman, J. (1996) J. Biol. Chem. in press.

19. Chen, C., and Okyama, H. (1987) Mol. Cell. Biol. 7, 2745-2752.

20. Kelley, M. J., Pech, M., Seuanez, H. N., Rubin, J. S., O’Brien, S. J., and Aaronson, S. A. (1992) Proc. Natl. Acad. Sci. USA 89, 9287-9291.

21. Cunha, G. R., Foster, B. A., Donjacour, A. A., Rubin, J. S., Sugimura, Y., Finch, P. W., Brody, J. R., and Aaronson, S. A. (1994) in (M.Motta and M.Serio, Eds.), pp. 45-57, Elsevier, Amsterdam.

22. Finch, P. W., Lengel, C., and Chedid, M. (1995) J. Biol. Chem. 270, 11230-11237.

23. Zhau, H. E., Hong, S.-J., and Chung, L. W. K. (1994) Int. J. Cancer 56, 706-714.

24. Chang, S.-M., and Chung, L. W. K. (1989) Endocrinol. 125:5, 2719-2727. 